\title{
Geographic Routing Protocol: A Review
}

\author{
Harminder Kaur ${ }^{1}$, Harsukhpreet Singh ${ }^{2}$, Anurag Sharma ${ }^{3}$ \\ ${ }^{1,2,3}$ Department of Electronics and Communication Engineering \\ ${ }_{1,2,3}$ CTITR, Jalandhar, India \\ ${ }^{1}$ harminder.kajal@gmail.com \\ 2harsukhpreet@gmail.com
}

\begin{abstract}
Mobile Adhoc Networks (MANETs) are the self-organized networks in which connections are not established for exchange the information. In MANET, there are major problems like scalability, dynamic topology, high mobility and routing. The network can be damaged due to the high mobility. Topology based routing can fail when there is a dynamic change in the network topology. To avoid these problems, Geographic routing is used. The geographic routing protocols are more efficient and scalable when there is a dynamic change in the network topology and when the mobility is high. In this paper, we have surveyed on the Hybrid Routing and Geographic routing protocol. The hybrid routing can be done into two ways i.e. greedy routing and face-2 algorithm or perimeter routing.
\end{abstract}

Keywords: MANET, Geographic Routing Protocol (GRP), Dynamic Topology, Position Based Routing.

\section{Introduction}

Technological advancement \& competition among mobile operators had contributed to wireless Networks growth reached 1700 million in 2010 and is predicted this growth will continue to rise. This growth can increase rapidly, can be more than 2500 million in 2015 [9]. Wireless networks can be divided into two parts such as infrastructure wireless networks and without infrastructure wireless networks. In infrastructure wireless networks there is a fixed infrastructure, mobile nodes can move from one base station to another base station, each base station has its own access point, where the communication is done through the access points between two base stations. While in infrastructure less or Adhoc networks there is no fixed infrastructure. Each and every node in this type of arrangement acts as a router as well as the network host. Mobile Ad-hoc Network (MANET) is a without infrastructure wireless network. Mobile ad hoc networks use multi-hop routing instead of static networks infrastructure to make available network connectivity. A MANET (Mobile Adhoc Network) is a wireless network in which network each mobile node communicates with each other without any infrastructure. In MANET, each node act as a router i.e. every node is responsible for transferring the information and for routing also [10]. Each MANET node may be prepared with different type of radio devices that have unstable transmission and receiving capabilities, and possibly operate on multiple asymmetric frequency bands links, which might result in heterogeneity in the radio capabilities to transfer its information. The key issues related to ad-hoc routing protocols are routing technique to be used that is unicast routing or multicast routing, dynamic network topology which changes when mobile nodes move from one base station to another base station as well as speed of mobile nodes[13]. Figure 1 shows the typical Mobile Adhoc Network. To avoid the problems 
of routing in dynamic topologies, the different routing protocols are used. The MANET routing is divided into three parts such as Proactive Routing, Reactive Routing, and Hybrid Routing. In proactive routing path is already known i.e. if a node wants to send the data the route for sending the data path is already known to the node. Each node maintains its routing table continuously. The routing table consists of source and destination address.

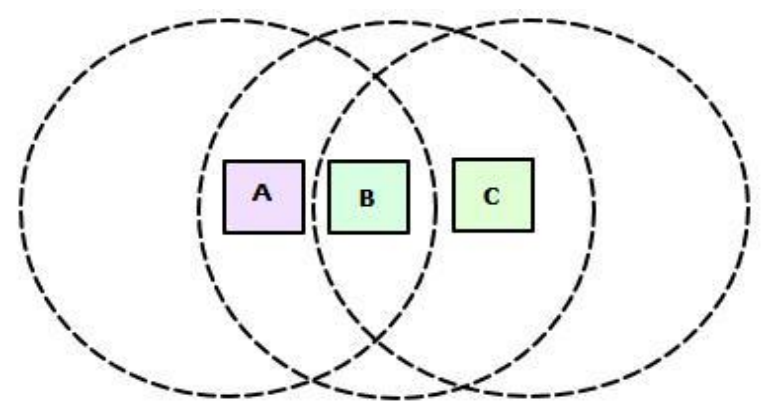

Figure 1: A Mobile Ad-hoc Network (MANET)

The proactive routing protocols are DSDV, WRP and so on. The limitation of this routing is more bandwidth utilization because the unused path occupies the available bandwidth of the network if the topology changes [12]. To avoid the limitation of proactive routing, reactive routing is used. In reactive routing, the route is only established when a node wants to send the data. As a result, it generates low traffic and routing overhead because the information is only sent when the user wants to send their data or information. The reactive routing protocols are AODV, TORA etc. The main advantage of reactive and proactive routing is less overhead and minimum power consumptions and less bandwidth requirement [9]. The drawback of using the reactive routing is that the delay will exist between the packets when transmitted, because routes in MANET are only established when needed. As the MANETs have a dynamic topology with the mobility of nodes, there will be a new route formation which results in increased overall network load [12]. The packets enrooted to the destination can be lost if the direction to the destination is changed. So, to avoid the problems in proactive and reactive routing, the hybrid routing is used. Hybrid routing is a combination of proactive routing and reactive routing. The path is established by using proactive routing and routing is done by reactive routing so it is called the hybrid routing. The hybrid routing is used to provide more efficiency and scalability of the network. Figure 2 shows the various routing protocols in MANET.

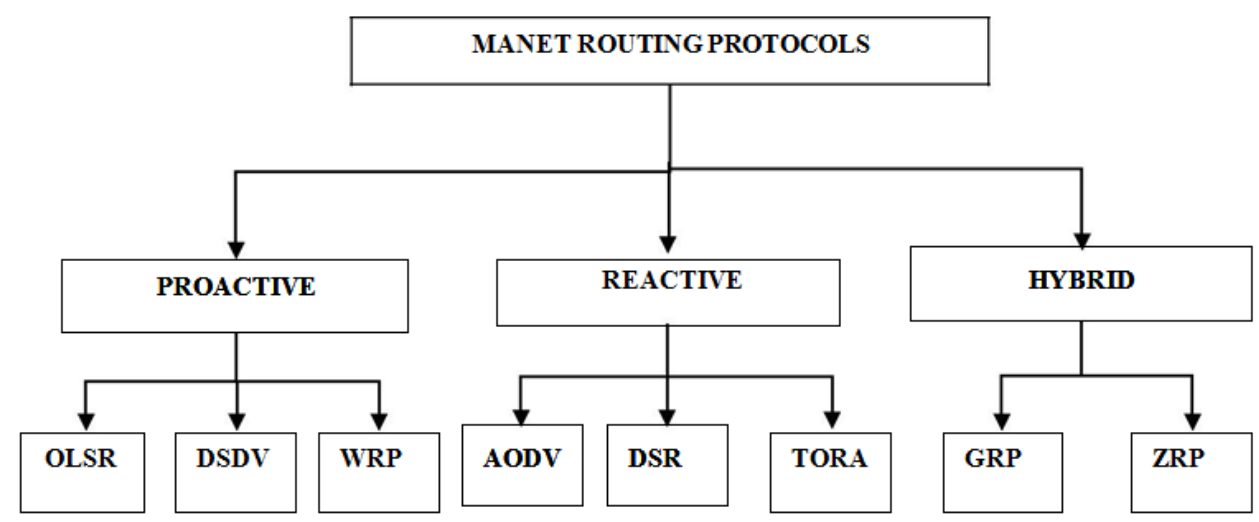

Figure 2. Routing Protocols in MANET 
In this paper, we have focused on hybrid routing and hybrid routing protocol such as GRP. The hybrid routing protocols are the combination of proactive routing and reactive routing, but some of the protocols are also using the concept of GPS (Global Positioning System) for transmitting the information. In GPS system, to send the information firstly the transmitter detects the position of the receiver and after detecting the location it sends the information, to use the GPS scheme node should be set with GPS receiver which is liable for detecting the position of the node in global organize system [17].

\section{Literature Survey}

Many of the researchers have been done in the field of MANET Networks, Routing in MANET and Geographic Routing. Mauve M. et al. [2001] had surveyed on greedy routing algorithms under geographical routing protocol in MANET. The geographic routing protocols are based on the greedy forwarding in which the data is sent to the nearest node of the target and neighbor node is selected by using different strategies of greedy forwarding. proposed Ad-hoc On-demand Distance Vector (AODV) routing protocol was intended for use by mobile nodes in an ad hoc network. It offered quick adaptation to dynamic link conditions, low processing and memory overhead, low network utilization and determines unicast routes to destinations within the ad hoc network. It used destination sequence numbers to ensure loop freedom at all times (even in the face of anomalous delivery of routing control messages), avoiding problems (such as "counting to infinity") associated with classical distance vector protocols [15]. Das et al. [2007] evaluated several routing protocols for mobile, wireless, ad-hoc networks via packet level simulations. The protocol suite included routing protocols specifically designed for ad-hoc routing, as well as more traditional protocols, such as link state and distance vector used for dynamic networks. The performance was evaluated with respect to the fraction of packets delivered, end-toend delay and routing load for a given traffic and mobility model. Authors observed that the new generation of on-demand routing protocols uses a much lower routing load. However the traditional link state and distance vector protocols provide, in general, better packet delivery and delay performance [4]. In IEEE 802.11 WLAN MAC - \& PHY Specifications [2007], the scope of this standard is to develop a medium access control (MAC) and physical layer (PHY) specification for wireless connectivity for fixed, portable, and moving stations within a local area. The purpose of this standard was to provide wireless connectivity to automatic machinery, equipment, or stations that require rapid deployment, which may be portable or handheld, or which may be mounted on moving vehicles within a local area. This standard also offered regulatory bodies a means of standardizing access to one or more frequency bands for the purpose of local area communication. This revision also specified technical corrections and clarifications to IEEE Std .802.11 for wireless local area networks (WLANS) as well as Enhancements to the existing medium access control (MAC) and physical layer (PHY) functions [5]. Anwar F. et al. [2008] described Worldwide Interoperability for Microwave Access (WiMAX) is a technology that bridged the gap between fixed and mobile access and offer the same subscriber experience for fixed and mobile user. The earliest version of WiMAX was based on IEEE 802.16 and was optimized for fixed and nomadic access, which is further extended to support portability and mobility based on IEEE 802.16e, also known as Mobile WiMAX. However, frequent topology changed caused by node mobility make routing in Mobile WiMAX networks a challenging problem. Authors focused upon those routing protocols especially designed for wireless networks, which studied and compared the performance of four ad-hoc routing protocols (AODV, DSR, 
OLSR and ZRP) for Mobile WiMAX environment under the assumption that each of the subscriber station had routing capabilities within its own network. Results found that ZRP and AODV protocols outperform DSR and OLSR [2]. Mamatha S. et al. [2010] proposed various deployment ways of mobile ad-hoc networks (MANETS) can have widely varying characteristics that have a greater impact on the behavior of different routing protocols created for these networks. Before directly deploying the applications in such environments, it was most important for developers to understand the potential quantitative behavior of the variations, challenges, capacity and implementation issues that supported their applications. Analytical models exist to describe the behavior of MANETS, but they were restricted to simplistic statistical models that represent either node mobility or link connectivity individually without considering the interplay of the two and other important aspects of MANETs [11]. Maghsoudlou A. et al. [2011] surveyed on the different face routing algorithms as well as different face routing strategies and greedy routing algorithms under the geographical routing protocol in MANET. Authors observed the geographic routing protocols are based on the greedy forwarding in which the data is sent to the nearest node of the target, but sometimes the data could be corrupted or lost or canceled if there is no foreigner node near to the target. Authors also proposed improve strategy to recuperate from this state and concluded that the most common strategy to recover from the state of the void is a face routing algorithm which uses the planner graphs [9]. Luo D. et al. [2011] analyzed the improved location based routing for Adhoc networks (IHLR) for overcome the problems of reactive routing protocols which combines the advantages of topology based routing and position based routing algorithms. The reactive routing protocols give the more routing overhead as compared to hybrid routing protocols. The IHLR protocol gave the minimal network delay and better throughput as compared to the reactive routing protocols [8]. Sharma S. et al. [2012] surveyed on Routing protocols and geographic routing protocols using GPS in MANET with their characteristics, functionalities, benefits, and limitations. Authors observed MANET is a dynamically changing network topology, in which the energy consumption increases with increase in mobility, so it is harder to achieve the better energy efficiency. Authors had purposed the protocol named as Energy saving Geographic Routing Protocol (ESGRP) using GPS which provides lower energy cost for effective routing solution [17].

Sharma V. et al. [2012] had surveyed on the different reactive routing protocols in MANET such as DSR, TORA, and AODV. Author's had been compared these three protocols and concluded that AODV protocol is best-suited protocol, as compared to other two protocols because network traffic load is less in AODV, is less than that of other protocols [19]. Khatkar A. et al. [2012] compared the Hybrid routing protocol and reactive routing protocols such as AODV and DSR by using different performance metrics such as Packet Delivery Ratio, End to End delay and Average Throughput. Authors concluded that hybrid routing protocols requires the minimal network delay and better PDR as compared to reactive routing protocols as well as the average throughput of hybrid routing protocols is better than that of reactive routing protocols [7]. Mittal P. et al. [2013] presented the comparison of MANET routing protocols i.e. GRP, AODV, OLSR and DSR on the basis of end-to-end delay, network load, retransmission attempts and throughput by using simulation tool OPNET modeler 14.5. Authors concluded that AODV and DSR perform better as compared to other protocols. The throughput of AODV and DSR is more as compared to other protocols and delay of AODV is lesser as that of other protocols [10]. Menon V. G. et al. [2013] analyzed the performance of the different geographic routing protocols in high mobility. Authors had been compared the performance of different geographic routing protocols on the basis of performance metrics and listed the merits and demerits of these protocols on the basis of their performance metrics. Authors had been discussed 
the different parameters involved for scheming and choosing a routing protocol [13]. Sharma V. et al. [2013] had compared the two reactive routing protocols i.e. AODV \& DSR in MANET network by using the real-time applications such as VoIP. Author had compared these two protocols by using different QoS parameters. Authors concluded that the AODV protocol is a best-suited protocol for reactive routing which give the minimal delay and performs better in a dense population of nodes [18]. Prasanth K. et al. [2014] had explained the Geo-cast routing protocols and their individuality such as scalability, message complexity, memory requirements and robustness. Authors proposed the temporally planned Routing Algorithm for Geo-cast routing, in which Location based routing uses the routing method based on flooding and without flooding network. The author concluded that in flooding location based routing; by using two methods the network maintain a multicast tree [16]. Wadhwa D. et al. [2014] compared different geographic routing protocol such as Location aided routing, Greedy perimeter stateless routing and Energy-aware geographic routing on the basis of performance metrics such as end to end delay and packet delivery ratio and energy utilization by using simulation tool NS2. Authors concluded that the geographic routing gives high packet delivery ratio, better energy utilization and better network lifetime as compared to other protocols when the topology changes dynamically and when the mobility is high [20].

\section{GEOGRAPHIC ROUTING PROTOCOL (GRP)- Analysis \& Discussions}

In the previous section, we have surveyed on the geographic routing protocol (GRP) and the methods of geographic or position based routing. The GRP routing protocol uses the concept of geographic routing for the exchange the information. Position based routing or geographic routing is used to eliminate the limitations of topology based routing. It gives the better performance in dynamic topologies because the packets are forwarded to its destination with respect to its position [17]. Each node determines its own position and for determining the position of the network node the different positioning schemes are used such as GPS, GPRS etc [16]. By using the concept of position based routing, the geographic routing protocols do not need to set up and to maintain the connections. In hybrid routing, the nodes do not require to store the routing tables and not to maintain the routing tables up to date for transmitting the information. It simply detects the location of the target node in the network and simply conveys the information from starting place to target. The way of transmitting the information in this protocol is based on the site information of the target and one hop neighbors.

There are two types of forwarding strategies in hybrid routing for conveying the information such as Greedy Forwarding and Face-2 Routing Perimeter [20] (figure 3).

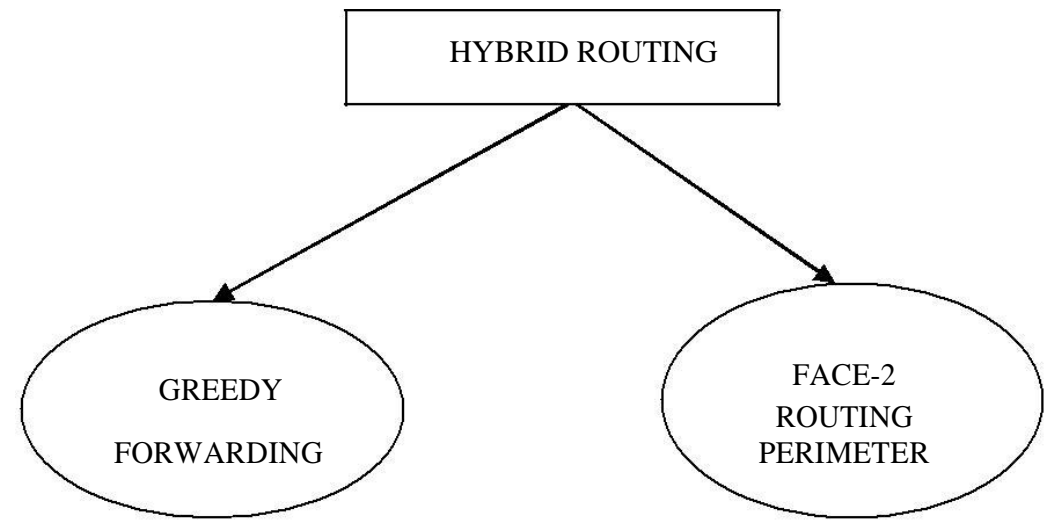

Figure 3. Types of Hybrid Routing 
In the Greedy forwarding method, the sender knows the estimated location of the receiver node. The message is forwarded to the nearest neighbor of the receiver node. The message is collected by the positioning scheme i.e. GPS. The in-between node receives the data to a neighbor two-faced in the way of the receiver node [12]. This process is continual until the data is reached at the receiver node. Each node in the network maintains its own table where the location of the each node is listed [20]. The main difficulty in greedy forwarding is to select the accurate neighbor node to send the data. For the selection of the neighbor node, the different routing strategies are used. The different routing strategies in the greedy forwarding are used defined as in terms of development, space and direction towards the receiver node [9]. There are three different routing strategies in greedy routing these are Most Forwarded within $\mathrm{R}$ (MFR), Nearest with Forwarded Progress (NFP) and Compass Routing. From the different strategies, a node can select to decide to which neighbor node the packet should be forwarded. These strategies are shown in Figure 4.

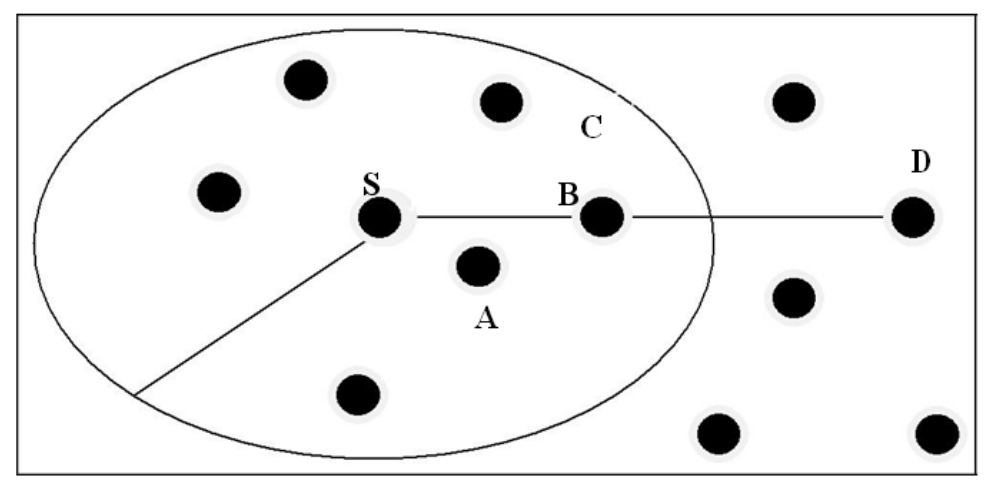

Figure 4. Greedy Routing Strategies [12]

From the figure 4 we can see that there are different strategies for a sender to send the information from source to a destination where $S$ donates source node and D donates destination node. The rounded area with $\mathrm{r}$ shows the coverage area or maximum range of the $S$. The main initiative is to send the information from $S$ to that node which is closest towards the $\mathrm{D}$. In the given example this node can be node $\mathrm{C}$ which can be closest towards the destination node within the coverage area of destination node D and this strategy is known as the Most Forwarded within R (MFR) and this strategy is tried to minimize the number hops to transmit the information from $S$ to D [13]. MFR is mostly used in that scenarios in which the packet does not adjust signal strength for the communication between $S$ and D. However, in which scenario the packet adjust or adapt its signal strength a different strategy is used i.e. Nearest with Forwarded Progress (NFP). In NFP, the message is transferred to the nearest neighbor of the sender which is closest to the destination. In the given example that node is A. If all nodes utilize NFP strategy, the collision of the packets during transmission can be reduced much. Another strategy used in greedy forwarding is Compass Routing, in which a sender node selects the neighbor closest to the straight line between the Source and Destination [12]. In the given figure, the compass routing node is node $\mathrm{B}$. This routing strategy is used to minimize the distance between the source to destination for the packet to travel.

When the packet reached at the node where it does not discover the neighbor node which is close to the destination with the greedy forwarding routing method then the second method of hybrid routing i.e. Face- 2 routing or Perimeter Routing is used to determine the destination address [20]. The problem in greedy routing is shown in Figure 5. In this figure the half circle around D has the radius of the distance between 
$\mathrm{S}$ and $\mathrm{D}$ and circle around $\mathrm{S}$ shows the range of the $\mathrm{S}$. From the figure we can say that there is no direct connection between $\mathrm{S}$ and $\mathrm{D}$, so the greedy forwarding fails here. To avoid the limitations of greedy forwarding methods another method is used which is known as the Perimeter Method or Face-2 Algorithm. In this type of routing technique, the packet is forwarded to the node with least backward progress if any of the nodes cannot find the forward path [7]. However, with this method the problem of looping packets is occurring, which does not occur in forward packet towards the destination with positive progress. The face- 2 algorithm is based on the planner graph traversal, in which a node does not need to store any additional or inadequate information. It proceeds to the greedy forwarding mode when the packet reaches to closer to the destination where the packet enters the improvement mode [13]. Figure 6 shows the planner graph traversal.

Planner graphs are the graphs with no intersecting perimeters. The information is transferred in an ad hoc network in which the nodes are vertices and the perimeter exists between two vertices if they are closer to communicate straight with each other. In the planner graph traversal, the packet is forwarded along the center of the face by using the right-hand rule: ahead the packet on the next hop by counter-clockwise from the perimeter on which it arrived. The line drawn between source $S$ and destination D intersects the perimeter along which a packet is to be forwarded. If this intersection is near to the destination then the additional intersection is encountered.

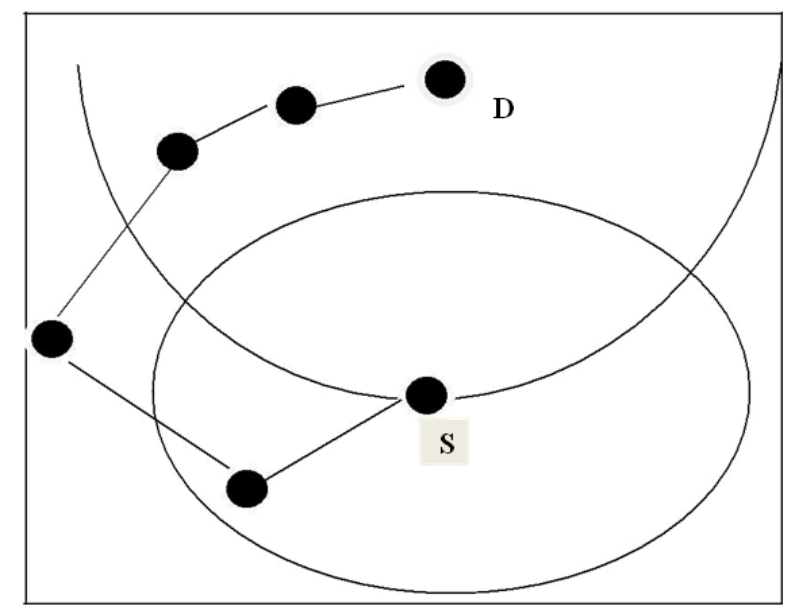

Figure 5. Greedy Routing Failure [12]

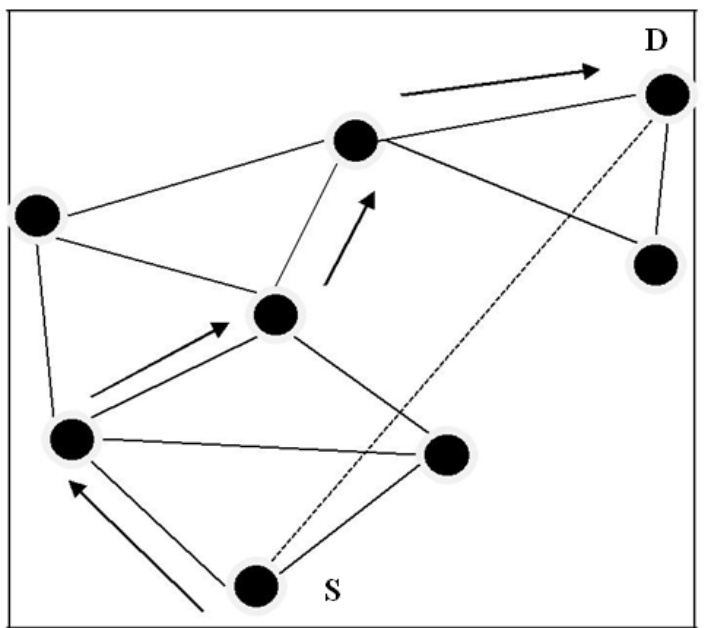

Figure 6. Planner Graph Traversal [12] 


\section{Conclusion}

In this paper, we have surveyed on geographic routing and geographic routing protocol (GRP). GRP protocol uses the concept of position based routing in which each node determines its position by using the different positioning schemes such as GPS and GPRS. By using the concept of position based routing, the routing node does not need to maintain its routing table continuously. In GRP, routing is done into two ways. In greedy forwarding, the information is sent to the nearest neighbor of the destination node by using the three different routing strategies. These routing strategies are used for the selection of the neighbor node. The other method of GRP routing is perimeter or face- 2 routing which uses the concept of planner graph transversal.

\section{References}

[1] Abolhasan M., Wysocki T. and Dutkiewic E., "A Review of Routing Protocols for Mobile Adhoc Networks,”ELSEVIER, Ad-hoc Networks (2004), vol. 2, pp-1-24.

[2] Anwar F., Azad S., Rahman A., Uddin M. M., "Performance Analysis of Ad hoc Routing Protocols in Mobile Wi-MAX Environment," IAENG International Journal of Computer sciences, 35:3, IJCS_35_3_13, 2008.

[3] Danson, D. P., Muknahallipatna, S., Stefanovic, M., "Performance of Mobile Ad-hoc Networks with Random and Predetermined Mobility Patterns using OPNET: Preliminary Results," proceedings of 32nd IEEE International Conference on Local Computer Networks, 2007 (LCN 2007), pp. 781-783.

[4] Das S. R., Castaneda R., Yan J., Sengupta R., "Comparative Performance Evaluation of Routing Protocols for Mobile, Ad hoc Networks," Proc. in 7th IEEE International Conference on Computer Communications and Networks, '98 , pp. 153 - 161, Vol., 2002.

[5] IEEE Computer Society, "IEEE Standard for Information technology-Telecommunications and information exchange between systems-Local and metropolitan area networks-Specific requirements, Part 11: Wireless LAN Medium Access Control (MAC) and Physical Layer (PHY) Specifications," IEEE Std. 802.11, 2007.

[6] IETF MANET Working Group, Mobile Ad Hoc Networks (MANET)", Working Group charter. http://www.ietf.org/html.charters/manet-charter.html ITU, "World Telecommunication Development Report 2002: Reinventing Telecoms,” March, 2002.

[7] Khatkar A and Singh Y., "Performance Evaluation of Hybrid Routing Protocols in Mobile Ad-hoc Networks", Advanced Computing \& Communication Technologies (ACCT-2012), DOI $10.1109,2012$.

[8] Luo D. and Zhou J, "An Improved Hybrid Location-Based Routing Protocol for Ad Hoc Networks", IEEE 2011.

[9] Maghsoudlou A.,Sthilaire M. and Kunz T., "A Survey on Geographic Routing Protocols for Mobile Ad hoc Networks," Carleton University, Systems and Computer Engineering, Technical Report SCE-11-03, October 2011.

[10] Mittal P.,Singh P. and Rani S., "Performance Analysis of AODV, OLSR, GRP and DSR Routing Protocols With Database Load in MANET," International Journal of Research in Engineering and Technology(IJRET), eISSN: 2319-1163, Volume: 02 Issue: 09 Sep, 2013.

[11] Mamatha S. and Sharma C., "Analyzing the MANET Variations, Challenges, Capacity and Protocol Issues," International Journal of Computer Science \& Engineering Survey (IJCSES), vol.1, No.1 pp. $14-21,2010$.

[12] Mauve M., Widmer J., and Hartenstein H,“A Survey on Position Based Routing in Mobile Ad Hoc Networks,” IEEE Network, November-December 2001.

[13] Menon V. and Prathap J., "Performance Analysis of Geographic Routing Protocols in Highly Mobile Ad Hoc Network," Journal of Theoretical and Applied Information Technology, Volume:54 No.1,10 August,2013.

[14] Murthy S., Garcia-Luna-Aceves J., "An Efficient Routing Protocol for Wireless Networks," ACM Mobile Networks and App. Journal, Special Issue on Routing in Mobile Communication Networks, pp.183-197, 1996.

[15] Perkins C., Royer E. B. and Das S., "Ad hoc On-Demand Distance Vector (AODV) Routing Internet Draft,” IETF Network Working Group, RFC 3561, pp.1-38, 2003.

[16] Prasanth K. and Sivakumar P., "Location Based Routing Protocol - A Survey," International Conference on Computer Communication and Informatics (ICCCI -2014), Jan. 03 - 05, 2014

[17] Sharama S. and Singh S., "A Survey of Routing Protocols and Geographic Routing Protocols Using

[18] GPS in MANET," Journal of Global Research in Computer Science(JGRCS), Volume 3, ISSN2229-371X, December 2012.

[19] Sharma V., Singh H., Kaur M. and Banga V., “ Performance Evaluation of Reactive Routing 
Protocols in MANET Networks using GSM based Voice Traffic Applications," OPTIK ELSEVIER, 2013.

[20] Sharma V., Kaur M. and Singh H. " A Survey on Reactive Adhoc Routing Protocols in MANET," CiiT International Journal of Wireless Communication,2012,DOI WC 042012001.

[21] Wadhwa D., Deepika, Kochher V.and Tyagi R., "A Review of Comparison of Geographic Routing

[22] Prptocols in Mobile Ad-hoc Network," Advance in Electronic and Electric Engineering, ISSN 22311297, Volume 4, pp. 51-58, 2014.

[23] Priyanshu and Maurya A., "SURVEY: Comparison Estimation of Various Routing Protocols in Mobile Ad-Hoc Network," International Journal of Distribute and Parallel Systems (IJDPS) Vol.5, No.1/2/3, May 2014.

[24] Karthikeyan.R, Sasikala.K and Reka.R., " A Survey on Position - Based Routing in Mobile Ad Hoc Networks," International Journal of P2P Network Trends and Technology (IJPTT) ,Volume 3,Issue 7,August 2013.

[25] Li Y., Yang Y., and Lu X., “ Routing Metric Designs for Greedy, Face and Combined-Greedy-Face Routing," INFOCOM, IEEE Proceedings, 2009.

[26] Ahmed, S., Alam, M. S., "Performance Evaluation of Important Ad-hoc Network Protocols,"

[27] EURASIP Journal on Wireless Communications and Networking, vol. 2006, issue 2, pp. 42-46.

[28] Barakovie, S., Barakovie, J., "Comparative Performance Evaluation of Mobile Ad-hoc Routing Protocols," proc. of the 33rd IEEE International Convention, MIPRO' 2010, pp. 518-513.

[29] Elizabeth, M., Toh, C., "A Review of Current Routing Protocols for Ad-hoc Mobile Wireless

[30] Networks," Ad-hoc Networks 2, pp. 1-22.

[31] Singh H, A, Sharma A, Sharma V , "Performance Optimization of DCF-MAC Standard Kaur using Enhanced RTS Threshold under impact of IEEE 802.11 n WLAN," Proc. of 2015 Fifth International Conference on Advanced Computing \& Communication Technologies (ACCT), IEEE, pp. 421-424, 2015.

[32] Sharma V., Malhotra J., Singh H, “ Performance Evaluation of MAC-and PHY-Protocols in IEEE 802.11 WLAN, " Proc. of springers, HPAGC-2011, 2011.

[33] Das S., Pucha H., and Hu Y., "Performance comparison of scalable location services for geographic ad hoc routing," INFOCOM 2005, vol. 2, pp. 1228-1239 vol. 2, March 2005.

[34] Elizabeth M., Toh, C., "A Review of Current Routing Protocols for Ad-hoc Mobile Wireless

[35] Networks," Ad-hoc Networks 2, pp. 1-22.

[36] Maihofer C., "A Survey of Geocast Routing Protocols," IEEE Communication Surveys, vol. 6, No.2, 2004

[37] Sharma V., Singh H., Malhotra J., “ Performance Analysis of IEEE 802.11 e (EDCF) and IEEE 802.11 (DCF) WLAN Incorporating Different Physical Layer Standards," Journal of The Institution of Engineers (India): Series B, Springer-Verlag, pp. 247-253, vol. 93, issue 4, 2012.

[38] Sharma V., Singh H., Kant S., " Challenging Issues in VANET Network and its Routing Algorithms- An Analysis," Proc. of Int. Conf. on Advances in Communication, Network, and Computing, DOI: 03.LSCS.2013.1, ACEEE, pp. 48-51, 2013. 
International Journal of Grid and Distributed Computing Vol. 9, No. 2 (2016) 